\title{
Síndrome de fatiga crónica en una adolescente de 15 años
}

\author{
A. Díaz-Caneja Greciano ${ }^{a, b}$, J.T. Rodríguez Sosa ${ }^{a}$, S. Aguilera Albesa ${ }^{a}$, \\ R. Sánchez-Carpintero ${ }^{\mathrm{c}}$ y C. Soutullo Esperón ${ }^{\mathrm{a}}$
}

aUnidad de Psiquiatría Infantil y Adolescente. Departamento de Psiquiatría y Psicología Médica. Clínica Universitaria. Universidad de Navarra. ${ }^{b}$ Unidad de Psiquiatría Infantil y Adolescente. Departamento de Psiquiatría y Psicología Médica. Clínica Universitaria (CUN). CUN-Madrid. cunidad de Neuropediatría. Departamento de Pediatría. Clínica Universitaria. Universidad de Navarra. España.

El cansancio y la falta de energía son síntomas muy frecuentes en niños y adolescentes. Su persistencia o aparición intermitente asociada o no a otros síntomas, sin causa fisica demostrable, debe hacer considerar la posibilidad de un síndrome de fatiga crónica. La falta de conocimiento de la enfermedad y un diagnóstico tardío pueden tener un impacto negativo en el desarrollo normal de los niños $y$ adolescentes afectados. El tratamiento debe estar basado en un programa de rehabilitación con aumento gradual de actividades y en la terapia cognitivo-conductual.

Palabras clave:

Síndrome de fatiga crónica. Diagnóstico. Tratamiento.

\section{CHRONIC FATIGUE SYNDROME IN A 15-YEAR-OLD GIRL}

Fatigue and lack of energy are frequent symptoms in children and adolescents. A diagnosis of chronic fatigue syndrome should be considered in children and adolescents who complain of chronic fatigue associated with other symptoms without a demonstrable physical cause. Lack of knowledge about this syndrome and late diagnosis may have a negative impact on the normal development of affected children and adolescents. Treatment should be based on a rehabilitation program with cognitive behavioral therapy and a gradual increase in activities.

Key words:

Chronic fatigue syndrome. Clinical diagnosis. Treatment.

\section{INTRODUCCIÓN}

La fatiga es un síntoma muy frecuente en los niños y adolescentes. En algunos casos puede ser debilitante y crónica, por lo que, una vez descartadas las distintas causas físicas que cursan con dicho síntoma, se debe considerar el diagnóstico de síndrome de fatiga crónica (SFC). El SFC afecta principalmente a adultos, aunque se ha descrito en niños y adolescentes ${ }^{1,2}$. El desconocimiento de la enfermedad por el pediatra, debido a su baja frecuencia, puede conducir a un diagnóstico tardío con un impacto negativo en el desarrollo normal de los niños y adolescentes afectados ${ }^{3}$.

Presentamos el caso de una adolescente que es derivada a nuestra Unidad de Psiquiatría Infantil y Adolescente con fatiga de larga evolución asociada a otros síntomas. Se describe el diagnóstico de SFC, el plan de tratamiento y su evolución inicial.

\section{Observación CLínICA}

Chica de 15 años que acude con sus padres a nuestra consulta derivada por el departamento de pediatría para valoración de ánimo bajo e intensa astenia de 3 años de evolución. El cuadro se inició con febrícula diaria, cansancio y cefalea, sin evidencia de enfermedad viral o sistémica. Acudieron a diversos especialistas y se le realizaron numerosas pruebas durante 3 años (analítica completa en varias ocasiones incluyendo anticuerpos antinucleares y una resonancia magnética cerebral) que no mostraron ninguna anormalidad. Se obtuvieron además serologías para virus de la hepatitis A, B, C, citomegalovirus, Chlamydia y Mycoplasma, que fueron negativas. La serología para el virus de Epstein-Barr (VEB) mostró una IgG positiva con IgM negativa.

A pesar de la negatividad de todos los estudios, la paciente presentó un empeoramiento progresivo de los síntomas iniciales, refiriendo, además, intensos dolores en

Correspondencia: Dra. A. Díaz-Caneja Greciano.

Unidad de Psiquiatría Infantil y Adolescente.

Departamento de Psiquiatría y Psicología Médica.

Gral. López Pozas, 10. 28036 Madrid. España.

Correo electrónico: adiazcaneja@hotmail.com

Recibido en enero de 2007.

Aceptado para su publicación en febrero en 2007. 
pequeñas y grandes articulaciones ante mínimos esfuerzos. Estos síntomas han afectado su funcionamiento normal, conduciéndole a un progresivo abandono de sus relaciones sociales, una mayor dependencia de sus padres para funciones básicas como vestirse o levantarse de la cama y a frecuente absentismo escolar. Los padres notaron que los síntomas se agudizaban tras episodios de amigdalitis, por lo que recientemente se realizó una amigdalectomía, sin observarse ningún cambio en la evolución del cuadro.

En el momento de la consulta, la paciente permanece habitualmente en casa, sin acudir al colegio debido al dolor intenso y al cansancio ante cualquier esfuerzo como levantarse de la cama.

Manifiesta que, debido a la situación actual, se encuentra con un humor triste, tiene sentimientos de inutilidad, angustia y sensación de bolo gástrico. Presenta dolores abdominales con pérdida de apetito y un patrón de sueño alterado. Apenas sale de casa, necesitando ayuda para vestirse y se encuentra triste porque según dice "no puedo hacer nada".

Entre sus antecedentes personales destaca la presencia de ansiedad por separación al inicio de la etapa preescolar hasta la edad de 6 años. La madre dice que era una niña tímida e introvertida, no hablaba de sus emociones o problemas y tenía dificultades para hacer amigas en el colegio. Siempre ha tenido un rendimiento académico alto.

La paciente comenzó sesiones individuales con una psicóloga con el objetivo de desarrollar estrategias para sobrellevar los síntomas. Sus padres manifiestan reiteradamente su preocupación por una posible causa física que explique los síntomas, a pesar de la negatividad de los estudios realizados.

$\mathrm{Al}$ acudir a nuestra unidad se realizó una nueva valoración pediátrica. La exploración física y neurológica incluido un balance muscular completo, fueron normales. Se repitió la analítica sanguínea que mostró de nuevo valores normales de transaminasas, enzimas musculares, hormonas tiroideas, hemograma completo, electrólitos, y metabolismo del hierro y calcio. La serología para el VEB demostró una IgG positiva con IgM negativa, similar a determinaciones anteriores en otros centros. Se añadieron otras serologías para Borrelia burgdorferi, Coxiella burnetii (fiebre Q), Salmonella typhi y paratyphi, que fueron negativas. La morfología en sangre periférica, las inmunoglobulinas y el fenotipo linfocitario fueron también normales, así como los anticuerpos para enfermedad celíaca realizados por antecedente de celiaquía en abuelo por parte de madre. Se estudiaron marcadores de enfermedades reumatológicas y autoinmunes, como anticuerpos antinucleares (ANA), factor reumatoide y crioaglutininas, con resultados dentro de la normalidad.

Durante la evaluación psicopatológica, se evidenció la presencia de síntomas depresivos y ansiosos como ánimo bajo, dificultad para concentrarse, sentimientos de inca- pacidad, apatía, astenia, anergia, ansiedad psíquica y somática, disminución del apetito e insomnio de conciliación.

En el Cuestionario de Capacidades y Dificultades (SDQ-Cas) ${ }^{4}$, se observó la presencia de problemas emocionales. El Minnesota Multiphasic Personality Inventory (MMPI) ${ }^{5}$ mostró que la paciente tiende al rechazo de los problemas y aparenta adecuación, control y efectividad. Utiliza mecanismos de negación de las dificultades emocionales con un escaso conocimiento sobre sus propias motivaciones y acciones. Puede expresar sus conflictos emocionales por medio de manifestaciones somáticas, no aceptando que la sintomatología existente pudiera tener un origen psicológico. En el Child Depression Inventory $(\mathrm{CDI})^{6}$, la puntuación indica la presencia de síntomas depresivos y en el Multidimensional Anxiety Scale for Children (MASC) ${ }^{7}$ obtiene puntuaciones altas en los ítems de fobia social y ansiedad psíquica.

El diagnóstico de SFC se consideró en esta paciente debido a la presencia de fatiga persistente, de más de 6 meses de duración, con ausencia de enfermedad sistémica y presencia de otros síntomas como mialgias, cefalea crónica, alteración del sueño y debilidad muscular.

\section{Tratamiento y curso clínico}

El plan de tratamiento para estos pacientes recomienda un programa de rehabilitación con un incremento gradual de actividades y regulación del ritmo del sueño con el objetivo a largo plazo de retornar al colegio. El apoyo psicológico está basado en la terapia cognitivo-conductual (modificación de pensamientos negativos y resolución de

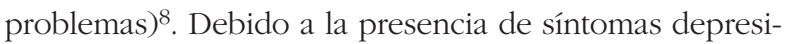
vos se comenzó, además, tratamiento con fluoxetina, inicialmente $10 \mathrm{mg} /$ día y tras 7 días se aumentó la dosis a $20 \mathrm{mg} /$ día con buena tolerancia de la medicación.

La evolución ha estado determinada por la dificultad para el cambio tanto por parte de los padres como de la paciente. Después del alivio inicial por la ausencia de una enfermedad sistémica, su posible presencia se ha cuestionado reiteradamente ante las continuas quejas de dolor y fatiga de la paciente durante cualquier actividad y la lenta mejoría de los síntomas. La familia ha dudado sobre si el programa terapéutico estaba empeorando la situación.

A pesar de estos problemas, la evolución está siendo favorable con una respuesta moderada al tratamiento combinado. En 3 meses, es capaz de tolerar más actividades y ha vuelto a reanudar el contacto con sus amigas. Todavía se queja de cansancio y dolor. Por decisión de la familia acude al colegio todos los días, aunque tiene problemas para completar las tareas escolares debido a problemas de concentración y memoria. La evaluación adaptada de su esfuerzo por parte de los profesores hace que se sienta menos presionada en el colegio. Continúa realizando el programa de actividades y acudiendo a las sesiones con la psicóloga. 


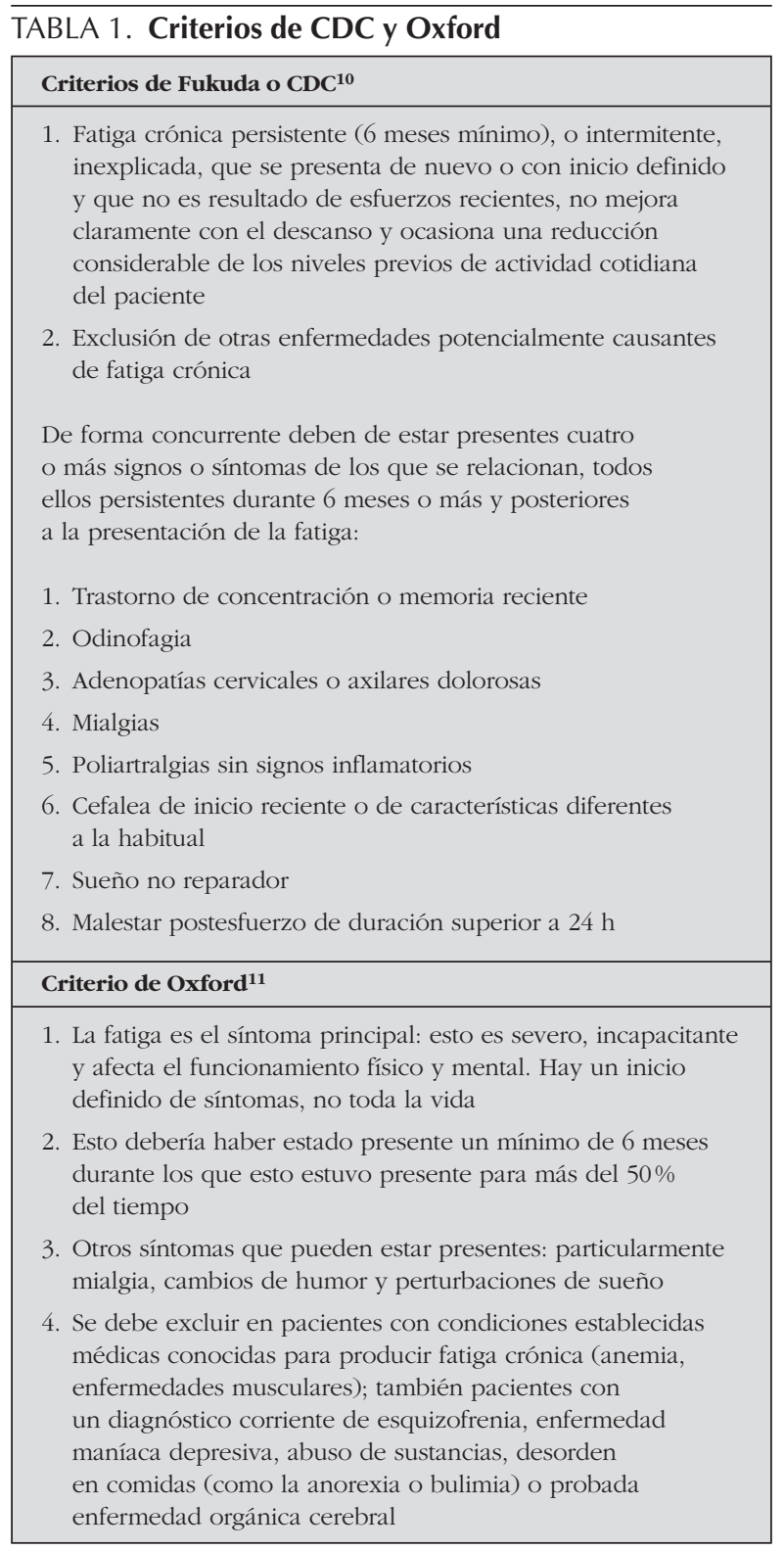

CDC: Centers for Disease Control and Prevention.

\section{DisCusión}

El SFC es un cuadro de etiología desconocida que cursa con fatigabilidad física y mental tras pequeños esfuerzos que no es explicable por una alteración médica o psiquiátrica. La prevalencia de SFC en adultos varía entre 0,2-2,6\%. En la población infantil y adolescente puede variar entre un $2 \%$ de niños y adolescentes en Estados Unidos y un 0,19\% de niños y adolescentes en el Reino Unido. Se sabe que es más frecuente en niñas como los trastornos del humor. Respecto a la posible mayor frecuencia del SFC en familias de nivel socioeconómico alto, puede ser un artefacto, ya que las familias con mayor nivel de educación y recursos tienen fácil acceso a las consultas especializadas ${ }^{2}$.
El SFC es de etiología desconocida y es éste uno de los motivos que hacen difícil el diagnóstico y el tratamiento. En dos terceras partes de los casos suele aparecer tras un episodio agudo infeccioso 9,10 . Algunas manifestaciones como dolores musculares y articulares generalizados, cefalea, dolor abdominal, diarrea, náuseas, faringitis, alteración en los patrones del sueño y comida suelen preceder a la fatiga y pueden mantenerse posteriormente durante la evolución de la enfermedad, contribuyendo a la confusión diagnóstica. En el estudio inicial de un SFC se deben descartar los principales trastornos que cursan con fatiga crónica, como anemia o enfermedades musculares. El conocimiento de esta enfermedad, junto con una exploración física y neurológica detalladas, permiten evitar pruebas complementarias costosas e innecesarias. Además, ayuda a aliviar la incertidumbre del paciente y su familia, que con frecuencia recorren un largo camino hasta llegar al diagnóstico, retrasando el inicio de la terapia más adecuada y favoreciendo el absentismo escolar y la aparición de psicopatología asociada ${ }^{3,11}$.

Se han desarrollado criterios diagnósticos aceptados internacionalmente ${ }^{12,13}$ (tabla 1). Ambas clasificaciones coinciden en que la duración de la fatiga debe de ser superior a 6 meses, sin embargo algunos expertos señalan que en niños y adolescentes es quizás un período de tiempo excesivamente largo. Los problemas emocionales, los cambios de humor y la comorbilidad de trastornos psiquiátricos son frecuentes en este grupo.

Respecto al tratamiento, estudios recientes han mostrado que un programa de ejercicio gradual y/o terapia cognitivo-conductual mejora los síntomas y las discapacidades asociadas en pacientes con $\mathrm{SFC}^{8,14,15}$. El pronóstico es mejor en niños que en adultos con una mejoría en un período de 2 años en el $75 \%$ de los casos $^{11}$.

Este caso de una adolescente con SFC muestra que el retraso diagnóstico puede agravar la sintomatología, favorecer la aparición de psicopatología y elevar el coste sanitario por consultas a múltiples especialistas, visitas a urgencias, y pruebas diagnósticas complejas, costosas, y algunas de ellas no exentas de riesgo. La comunicación rápida y fluida entre los Servicios de Pediatría y Psiquiatría Infantil y del Adolescente en estos casos, y la instauración precoz de la terapia de rehabilitación y cognitivo-conductual pueden reducir las complicaciones a largo plazo.

\section{Bibliografía}

1. Garralda ME, Rangel L. Annotation: Chronic fatigue syndrome in children and adolescents. J Child Psychol Psychiatry. 2002; 43:169-76.

2. Garralda ME, Chalder T. Annotation: Chronic fatigue syndrome in childhood. J Child Psychol Psychiatry. 2005;46:1143-51.

3. Sankey A, Hill C, Brown J, Quinn L, Fletcher A. A Follow-up Study of Chronic Fatigue Syndrome in Children and Adolescents: Symptom Persistence and School Absenteeism. Clin Child Psychol Psychiatry. 2006;11:126-38. 
4. Goodman R. The Strengths and Difficulties Questionnaire: A Research Note. J Child Psychol Psychiatry. 1997;38:581-6.

5. Hathway SR, McKinley JC. Inventario Multifásico de Personalidad de Minnesota-2 (MMPI-2). Manual. Adaptación Española. En: Avila-Espada A, Jiménez Gómez F, editores. Publicaciones de psicología aplicada. Madrid: TEA ediciones, S.A.; 1999.

6. Kovacs M. The Children's Depression, Inventory (CDI). Psychopharmacol Bull. 1985;21:995-8.

7. March JS, Parker JD, Sullivan K, Stallings P, Conners CK. The MASC (Multidimensional Anxiety Scale for Children). J Am Acad Child Adoles Psy. 1997;36(4):554-65.

8. Chalder T. Chronic fatigue syndrome. En: Graham P, editor. Cognitive behaviour therapy for children and families. Cambridge: Cambridge University Press; 2004. p. 385-402.

9. Garralda E, Rangel L, Levin M, Roberts H, Ukoumunne O. Psychiatric Adjustment in Adolescents with a History of Chronic Fatigue Syndrome. J Am Acad Child Adolesc Psychiatry. 1999;38:1515-21.
10. Carter B, Edwards J, Kronenberger W, Michalczyk L, Marshall G. Case control study of chronic fatigue in paediatric patients. Paediatrics. 1995;95:179-86.

11. Rangel L, Garralda ME, Levin $M$, Roberts $H$. The course of chronic fatigue syndrome. J R Soc Med. 2000a;93:129-34.

12. Fukuda K, Strauss S, Hickie I, Sharpe M, Dobbins J, Komaroff and the international Chronic Fatigue Syndrome Study Group. The chronic fatigue syndrome: a comprehensive approach to its definition and study. Ann Intern Med. 1994;121:953-9.

13. Sharpe MC, Archard LC, Banatvala JE, Broysiewicz L, Clare A, David A, et al. A report - chronic fatigue syndrome: Guidelines for research. J R Soc Med. 1991;84:118-21.

14. Wright B, Ashby B, Beverley D, Calvert E, Jordan J, Miles J et al. A feasibility study comparing two treatment approaches for chronic fatigue syndrome in adolescents. Arch Dis Child. 2005;90:369-72.

15. Chalder T, Tong J, Deary V. Family focused cognitive behaviour therapy for chronic fatigue syndrome. Arch Dis Child. 2002;86:95-97. 